\title{
Homeostatic Determinate Systems and Thermodynamics of Selforganization and Evolution of Material World
}

\author{
Lev E. Panin \\ Scientific Research Institute of Biochemistry, Siberian Branch of RAMS, Novosibirsk, Russia \\ E-mail: ibch@soramn.ru
}

Received January 31, 2011; revised March 3, 2011; accepted March 7, 2011

\begin{abstract}
The paper considers new approaches to system analysis of natural phenomena in physics, chemistry and biology. It lays the foundation of the homeostatic determinate systems theory that allows revealing the mechanism by which the basic principle of natural science, determinism, is being realized. Evolution of the material world is represented as inevitable and continuous growth of orderliness (negentropy) based on transition from one type of determinate systems to another. Increasing negentropy is shown to be closely associated with continuous accumulation of information, which determines the natural diversity in physics, chemistry and biology.
\end{abstract}

Keywords: Homeostatic Determinate System, Thermodynamics, Evolution

\section{Introduction}

The emergence of modern material world in its developed form is still an unsolved physical problem.

To emphasize the connection of physical laws of the evolving Universe with the emergence of Homo sapiens, the anthropic principle has been formulated. It reads thus: "We see the Universe as it is, because if it were different, we would not be here and we could not observe it" [1]. The anthropic principle has weak and strong versions. According to the weak anthropic principle, rational beings may appear only in some regions of the giant expanding Universe, which have space and time constraints. If the Big Bang occurred tens billion years ago, it means that this time was required for our galaxy to emerge and then for intelligent life to come into existence. The strong anthropic principle states that there are millions of different regions of the Universe or even the Universes, whose physical laws or initial conditions prevent the emergence of complex biological objects and intelligent life. However, in some regions of the Universe or in different Universes there formed such a set of physical laws that could lead to the appearance of rational beings. At least, our existence allows us to think so. "We see the Universe as we see it because we exist" [1]. To strengthen the anthropic principle, it should be noted that this process was determinate and inevitable rather than stochastic. However, it is necessary also to reveal the initial condi- tions and the mechanism that allowed the Universe to turn from its emergence to progressive evolution resulting in the origin of intelligent life.

The development of material world toward complexity and increasing natural diversity violates the second law of thermodynamics and makes it necessary to investigate nonequilibrium processes that may give rise to orderlyness. Now these problems are considered by synergetics. In this connection, H. Haken wrote: "In physics there is a notion of "concerted effects"; however, it is applied mainly to the systems in thermal equilibrium. I felt that I should introduce a term for consistency in the systems far from thermal equilibrium... I wished to emphasize the need for a new discipline that will describe these processes... Thus, synergetics can be considered as a science dealing with the phenomenon of self-organization" [2].

Among classical and well studied examples of selforganization in nonequilibrium systems are the Benard instability, Belousov-Zhabotinsky reaction, coherent radiation of atoms in lasers, various chemical waves, etc. [3]. All these phenomena belong to dissipative structures, which "are an amazing example demonstrating how nonequilibrium may be a source of order" [4].

Thus, now the self-organization processes are considered as a genesis of new structures and new behavioral patterns in the far-from-equilibrium open systems [5].

Probably, this is true if self-organization is considered 
only within the concept of dissipative structures. However, self-organization of matter in the course of its progressive and stable evolution is a much more complicated process. We should understand how the nature came from absolute chaos and maximum entropy to the processes of sustainable self-organization.

\section{System Analysis of Self-Organization Mechanisms in Material World, Homeostatic Determinate Systems in Physics and Chemistry}

Let us consider the simplest example of matter (atoms and molecules) self-organization. This is the formation of hydrogen molecules from hydrogen atoms.

If an atom has unpaired electron in its outermost electron level (shell) and such atoms approach each other, their electron waves overlap to form a new wave, which unites two atoms. Thus the covalent bond forms. Each hydrogen atom has one s-electron. When such atoms are approaching each other, an electron pair forms; this generates a single standing wave that combines two hydrogen atoms into a molecule. The formation of a covalent bond is advantageous in energy terms. Thus, collectivezation of electrons in a hydrogen molecule $\left(\mathrm{H}_{2}\right)$ increases their potential energy by $1.5 \mathrm{eV}$ and decreases the kinetic energy by $7.5 \mathrm{eV}$. Full energy of the interference of two waves becomes $6 \mathrm{eV}$ lower. The deformation of electron clouds makes energy transformations more complicated (Table 1).

One may see from Table 1 that the overall energy gain amounts to $4 \mathrm{eV}$; however, strength of the chemical bond between hydrogen atoms depends primarily on releasing $7.5 \mathrm{eV}$ at interference of two waves. Strength of a covalent bond in the hydrogen molecule is $104 \mathrm{kcal} / \mathrm{mol}$.

The formation of hydrogen molecule from two atoms is thermodynamically advantageous. Total energy of such system is lower by $4.0 \mathrm{eV}$. A new stable structure with new properties has formed. This is the process of self-organization. The work related with synthesis of $\mathrm{H}_{2}$ molecule was performed due to energy supplied from the

Table 1. Energy transformations at the interaction of two hydrogen atoms, eV.

\begin{tabular}{cccc}
\hline & \multicolumn{3}{c}{$\begin{array}{c}\text { Components of electron energy } \\
\text { of } \mathrm{H}_{2} \text { molecule }\end{array}$} \\
\cline { 2 - 3 } Energy & $\begin{array}{c}\text { Energy of } \\
\text { interference }\end{array}$ & $\begin{array}{c}\text { Energy of } \\
\text { modification, } \\
\text { etc. }\end{array}$ & \\
\hline Kinetic & -7.5 & +11.5 & +4.0 \\
Potential & +1.5 & -9.5 & -8.0 \\
Full & -6.0 & +2.0 & -4.0 \\
\hline
\end{tabular}

environment. Such system has a higher orderliness (negentropy).

Changes in the amount of information caused by appearance of the new system (structure) can be calculated quite easily.

In atomic hydrogen, the probability that electron occupies one of allowed energy levels is rather high. A minimum of information is connected with such electrons. When each pair of electrons forms a new hybrid wave, this abruptly decreases the number of degrees of freedom, and their behavior becomes determinate. In a new wave, each electron may stay only in one of two allowed states, which differ in their spin $(+1 / 2$ or $-1 / 2)$. According to Shannon's equation,

$$
-H=\sum p_{i} \log p_{i}
$$

the amount of information connected with such electron increases and depends on the quantity $1 / 2 \times \log 1 / 2$. For each pair of electrons this will be equal to $\log 1 / 2$. Multiplying this quantity with the number of hydrogen molecules in the given gas volume gives the total amount of information connected with electrons. This value corresponds to an increase of negentropy upon transition from atomic to molecular hydrogen.

Note that the process moves towards increasing negentropy and may proceed under the natural conditions. This is how the time's arrow forms, moving towards decreasing entropy in the nature, i.e., from chaos to matter self-organization due to delivery of energy from the environment. In biological objects, the source of external energy for various biosynthetic reactions is the oxidation processes related with decomposition of rather complex organic compounds (carbohydrates, lipids, and partially proteins). This is why E. Schrodinger believed that we are fed with negative entropy, thus being "the organization sustained by derivation of orderliness from the environment" [6].

The formation of hydrogen molecules from hydrogen atoms proceeds as a determinate process. The structure of hydrogen atom is very simple: a proton with an electron revolving around it. In the atomic state, hydrogen is a gas with a high level of entropy (low level of negentropy). Its atoms, colliding with each other, excite electrons, thus making them to occupy different energy levels and sublevels. Such electrons have a large number of degrees of freedom. However, the most probable is the state when electron occupies the lowest energy level (1s). For such a gas, temperature elevation serves as a specific signal interacting with the memory elements (electromagnetic forces). As kinetic energy of the gas molecules increases to a certain value, atoms having electrons at the 1s-level will interact with each other to form hydrogen molecules. Therewith, electrons form a new hybrid wave. 
However, more than one decision can be implemented at the formation of hybrid waves. According to the first decision (and action program), both electrons form a "convenient" wave; at that, they belong simultaneously to both hydrogen atoms. The second decision (and action program) implies the formation of "inconvenient" wave. In this case, a crest and a trough of the hybrid wave are separated by the rest zone (Figure 1). Such wave is thermodynamically unstable. It is destroyed, and then a decision is made to form a "convenient" wave.

It seems reasonable to describe such mechanisms of the material world self-organization using the concept of homeostatic determinate systems [3].

The concept of homeostatic determinate systems is fundamental for synergetics. It provides deep insight into physical meaning and genesis of the hierarchy of instabilities in self-organizing homeostatic systems, into the nature of interrelations between instabilities and order parameters [3,7].

Under homeostatic determinate systems we understand such systems where the eventual (actual) result of an action is predicted (determined) via the interaction of signals specific for the given system with its memory elements. Structurally such systems include determinate synthesis, choice of an adequate action program, its outcome, and feedback closed on results-of-action acceptor (Figure 2).

The most complex element of any homeostatic determinate system is the determinate synthesis. Here the dominant motivation, conditional (environmental) and causal (triggering) afferentation interact with the memory elements of the system. This results in decision making and choosing a termodinamic determined action program. Information on the achieved result goes to results-of-action acceptor (feedback), where the anticipated and actual results are compared. If the goal is not reached, i.e., the actual result does not fit the anticipated one, homeostatic determinate systems switch over to another programs due to changes in the decision made.

This mechanism of the material world self-organization radically differs from self-organization in dissipative

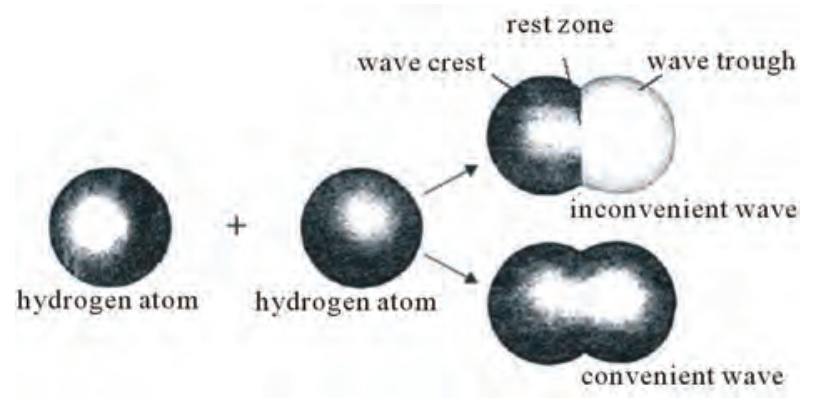

Figure 1. Programs used for the formation of covalent bonds in hydrogen molecules.

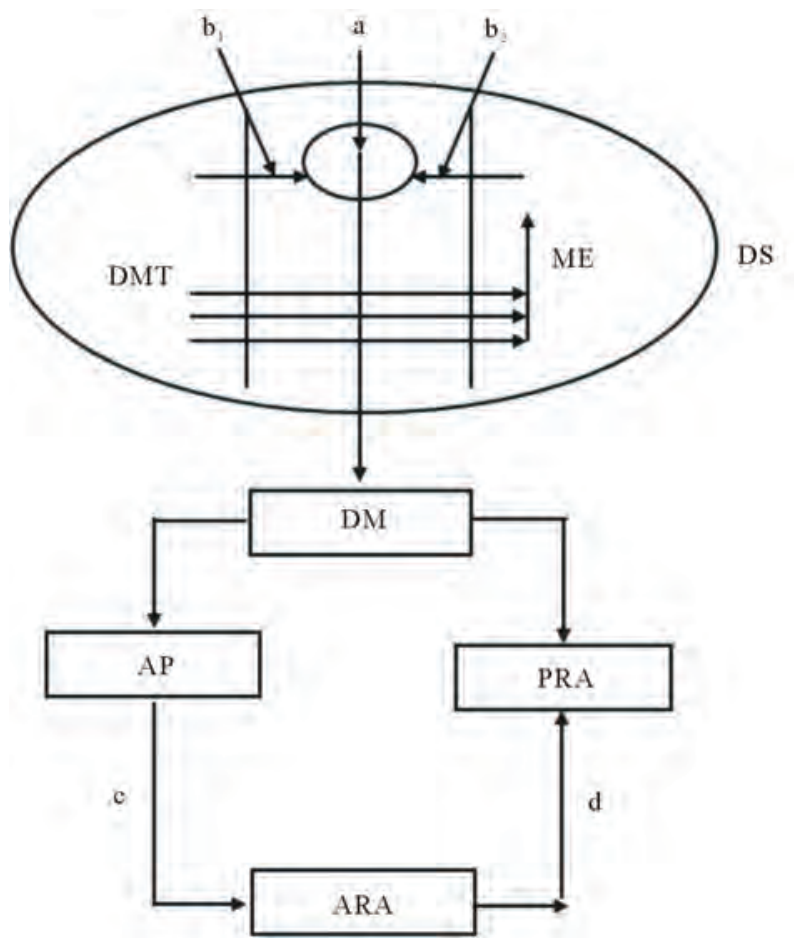

Figure 2. A schematic diagram of homeostatic determinate system: DMT - dominant motivation: system in a condition of thermodynamic balance; ME - memory elements: metal, covalent and hydrogen bonds, electrostatic and hydrophobic interactions; a - trigger signaling: specific signals able to interact with ME (mechanical load, tem- perature, pressure, electric discharges, etc.; $b_{1}$ and $b_{2}$ - circumstantial signaling: additional signaling accompanying the main one, for example, an excited environment; DM - decision making: transition of crystal to a new level of systemic organization; AP - action program: formation of new bonds, which change negenthropy (information) or enthropy; ARA actual results of action: emergence of a new system in one of energetically allowed state; PRA - predicted result of action: emergence of a new system in the energetically stable state; DS - determinate synthesis, decision-making process; $c$ and $\mathrm{d}$ - straight line and feedback.

nonequilibrium systems, where dissipative structures emerge due to reinforcement of the corresponding fluctuations. The appearance of such structures was called by I. Prigogine as "orderliness through fluctuations" [4]. Such mechanism of self-organization is abundant in the nature, but it cannot underlie the progressive evolution of material world, which is a stable and irreversible process. The nature has chosen the way of self-organization via the formation of homeostatic determinate systems.

In physical determinate systems, the system-forming factors are represented by four types of interaction:

- strong, or nuclear interaction (force) acts only between hadrons that form the atom nucleus (proton, neutron), and does not depend on electric charge of the interacting particles; 
- weak interaction (force) acts between leptons (electron), between hadrons, between leptons and hadrons, and does not depend on the charge of interacting particles;

- electromagnetic interaction (force) acts between electrically charged particles. With unlike charges it shows up as attraction; with like charges, as repulsion;

- gravitational interaction (force) acts between all the particles without exception and shows up as attraction. Since elementary particles have small masses, the role of gravitational interaction between them is negligible.

Due to the four type interactions, atoms of all chemical elements exist as systems, which are so stable under natural conditions that we can investigate their properties and use them. It can be stated that each chemical element (atom) is a unique determinate system with unique properties.

The forces serve as memory elements in these systems and determine their stability.

Strong interaction exists between quarks that form protons and neutrons. It is performed via the exchange of gluons $\left(G^{0}\right)$. Weak interaction is performed with $W^{ \pm}$and $Z^{0}$ bosons. They play an essential role in the formation of nuclei. Weak proton-electron interaction may be accompanied by the exchange of vector boson $W^{+}$or by the exchange of neutral $Z^{0}$ boson. Electromagnetic interacttion is accompanied by the exchange of photons $(\gamma)$. Gravitational interactions result from the exchange of gravitons. This force manifests itself at the interaction of large mass.

The exchange statuses form feedforwards and feedbacks in the functional pairs. The main constituting elements of atoms are presented in Tables $\mathbf{2}$ and 3. Some of them have a complicated structure.

Table 2. Elementary particles constituting the atoms.

\begin{tabular}{|c|c|c|c|c|c|c|}
\hline \multirow[b]{2}{*}{ Particle } & \multirow[b]{2}{*}{ Charge } & \multirow[b]{2}{*}{ Weight* } & \multicolumn{3}{|c|}{ Type of interaction } & \multirow[b]{2}{*}{ Stability } \\
\hline & & & Strong & $\begin{array}{l}\text { Electro- } \\
\text { magnetic }\end{array}$ & Weak & \\
\hline Proton & +1 & 1 & + & + & + & High \\
\hline Neutron & 0 & 1 & + & + & + & Low \\
\hline Electron & -1 & $1 / 1800$ & - & + & + & High \\
\hline Photon & 0 & 0 & - & + & - & High \\
\hline Neutrino & 0 & 0 ? & - & - & + & High \\
\hline $\begin{array}{l}\text { Charged } \\
\text { pi-meson }\end{array}$ & \pm 1 & $1 / 7$ & + & + & + & $\tau=10^{-8} \mathrm{~s}$ \\
\hline $\begin{array}{c}\text { Neutral } \\
\text { pi-meson }\end{array}$ & 0 & $1 / 7$ & + & + & + & $\mathrm{T}=10^{-16} \mathrm{~s}$ \\
\hline
\end{tabular}

* - in units of proton weight.
Table 3. Structure of protons and neutrons.

\begin{tabular}{cccc}
\hline Particle & \multicolumn{3}{c}{ Quarks } \\
\hline Neutron $\mathrm{n}^{0}$ & $\mathrm{U}^{+2 / 3}$ & $\mathrm{~d}^{-1 / 3}$ & $\mathrm{~d}^{-1 / 3}$ \\
Proton $^{+1}$ & $\mathrm{~d}^{-1 / 3}$ & $\mathrm{U}^{+2 / 3}$ & $\mathrm{U}^{+2 / 3}$ \\
\hline
\end{tabular}

The proton sounding with a high-energy neutrino beam reveals a very complicated pattern. Proton no longer looks as a simple combination of some quarks and gluons. A high activity is observed inside the proton, caused by its interaction with vacuum. It turns out that proton comprises a large number of quarks, antiquarks and gluons. Three "valence" quarks are hardly distinguished, slightly contributing to the mass of proton (see Figure 3).

Three "valence" quarks are hardly discernible among numerous gluons and quark-antiquark pairs (from Perkins [8]).

Atom as a system possesses all the features of determinate systems:

1) Memory elements (four type forces) that are involved in the interaction with external signals specific for the atom;

2) Decision making that chooses a certain action program under disturbance;

3) Action program that changes the nature of interacttion between the structure-forming particles;

4) Result of action - the atom acquires new properties.

To provide stability of the system, feedback should be introduced. The feedback will close between the actual and predicted results of action (PRA), which are the stable state of the exchange pairs implementing the four types of interaction. The interaction energy in the pairs should be minimal. In determinate systems they function as the results-of-action acceptor.

If stable interaction is not observed in the exchange pairs, the action program is corrected. Therewith, spontaneous transitions may occur in the pairs; however, they may occur also with virtual pairs, i.e., the particles-anti-parti-

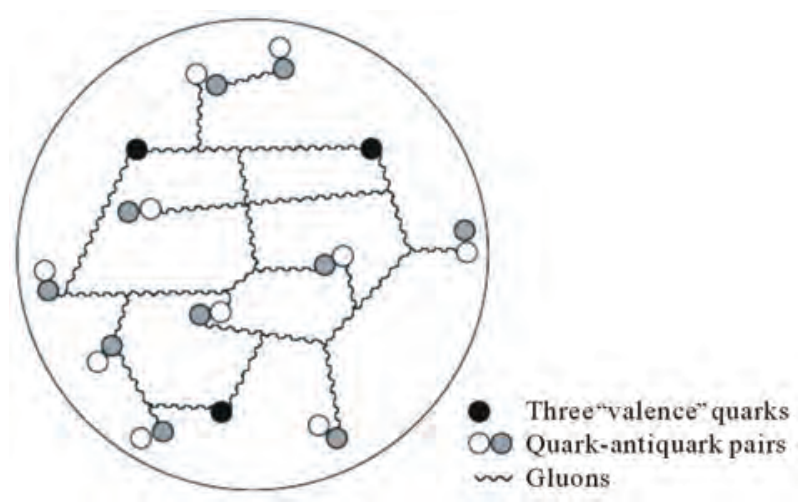

Figure 3. Proton observed with the radiation providing resolution on ca. $0.01 \mathrm{fm}\left(10^{-15} \mathrm{~cm}\right)$. 
cles borrowed from vacuum. It became possible to oppose the principle of determinism against the relativistic principle underlying the behavior of elementary particles.

Functional pairs in atoms serve as the results-of-action acceptors; therewith, the formation of a stable state is determined by the interaction of atom with the environment (vacuum) involving the virtual particles. Hence it follows that the environment (physical vacuum) is most essential for stable existence of any atoms and related quantum transformations. Atoms, similar to living objects, are the open systems, which continuously exchange mass, energy and information with the environment, i.e., with the quantum vacuum.

The concept of determinate systems is a new instrument for the analysis of functional states of atoms and a new conceptual apparatus (language) for their description. They make it possible to consider statistical (stochastic) processes in quantum physics as determinate, i.e., as the processes based on specific causal connections and quite predictable results.

Einstein wrote: "I still believe in a possibility of constructing such model of reality, i.e., the theory that expresses the objects themselves, but not only the probabilities of their behavior" [9].

The formation of all atoms of the periodic table of elements is considered in special literature [10]. Elementary particles and primary atoms present in the environment served as a construction material for the process. Thus, the presence of hydrogen and its isotopes (deuterium and tritium) enabled synthesis of helium atoms in the hot Universe:

$$
{ }_{1}^{2} \mathrm{H}+{ }_{1}^{3} \mathrm{H} \rightarrow{ }_{2}^{4} \mathrm{He}+\mathrm{n}_{0}+17.6 \mathrm{MeV} .
$$

These processes take place in stars. In our Solar system they play a unique role. This reaction, releasing a tremendous amount of heat, provided energy for evolution of material world on the Earth and origin of life, which is related with increasing chemical diversity.

The appearance of unique properties of the chemical elements due to increase in their weight and complication of their structural organization can be illustrated by example of carbon and oxygen.

Carbon has two electrons at the first energy level and four electrons at the second level, two of them occupying s-sublevel and the other two, p-sublevel. This element is very essential for all biological objects. Oxidizing, carbon acquires the valence +4 , reducing, -4 . This feature allows carbon atoms to interact with each other and form long aliphatic chains. Hydrocarbons are the most abundant among them. When interacting with metals, carbon is electronegative, with nonmetals it is electropositive. The most part of carbon in the earth crust is fixed as the limestone, $\mathrm{CaCO}_{3}$, and dolomite, $\mathrm{MgCa}\left(\mathrm{CO}_{3}\right)_{2}$. Coal, oil and gas are also the carbon-containing compounds.

Pure carbon forms such dissimilar crystals as graphite and diamond. The difference depends on the type of carbon packing in the crystals. In graphite, carbon forms an ordered plane structure. Plane crystals weakly interact with each other and readily desquamate. In diamond crystals, carbon forms three-dimensional structures, with strong forces of intermolecular interaction operating here. Such packing allows diamond of any size to be considered as a single molecule. Carbon-based chemical compounds make the foundation for all biological objects.

Oxygen is a gas, its atom is composed of eight neutrons, eight protons and eight electrons; the atomic weight is 15.994. It has three isotopes $\left({ }^{16} \mathrm{O},{ }^{17} \mathrm{O}\right.$ and $\left.{ }^{18} \mathrm{O}\right)$. Oxygen may form compounds (oxides) with each of chemical elements, including inert gases. It is the only gas that exhibits paramagnetic properties. Its involvement in the oxidation processes (combustion) is an important source of heat. In biological objects, oxygen sustains the processes of slow oxidation of organic substances, supplying us with a necessary amount of energy. These properties of oxygen are also vital for the developed form of life existing on the Earth.

Thus, formation of new physical determinate systems (atoms) proceeded until the emergence of unstable radioactive elements. Beyond the $104^{\text {th }}$ element of periodic table of chemical elements, all atoms were very unstable. Evolution of material world in this direction became impossible.

Further increase in natural diversity should be related with another homeostatic determinate system. Here, the memory elements were represented by new forces: covalent and hydrogen bonds, hydrophobic and electrostatic interactions. This was a fundamental change in the evolution of material world. It was related with the active formation of chemical determinate systems. Not only the memory elements of these systems have changed, but also their action program. The ability of electrons to form different hybrid waves (action program) became of crucial importance.

Example 1. A carbon atoms has four unpaired electrons in its outermost electron shell, which form four lobe waves (Figure 4(a)). Each of the waves may interact, for example, with the sphere wave of hydrogen atom. There are four hybrid lobe waves formed by four pairs of electrons, which are typical of the methane $\left(\mathrm{CH}_{4}\right)$ molecules. Spatially such molecule is a tetrahedron.

Example 2. The formation of ethylene. In this molecule, two carbon atoms are coupled with a double bond, the remaining free bonds being saturated with hydrogen atoms. This occurs as follows. Each carbon atom uses two octuple waves and one sphere wave to form three lobe waves arranged in the molecule plane. Both carbon 
atoms form an internal stable hybrid wave from two lobe waves. In such a way the first pair of electrons is used. The second pair of electrons in a double bond forms the external wave at interference of two octuple waves. In the process, two wave crests merge at the top, and two wave troughs merge at the bottom, or vice versa. The external wave covers carbon atoms like a jacket (Figure 4(b)). Double bond has a high strength, so the distance between carbon atoms here is shorter as compared to a single bond. Double bond makes the molecule more rigid, however, enhancing its reactivity. The molecule can sacrifice one of these bonds to interact with other atoms or chemical groups, still retaining its atoms bound.

Example 3. In the $\mathrm{H}_{2} \mathrm{O}$ molecule, bonding involves two unpaired p-electrons of the oxygen atom. Their orbitals make an angle of $90^{\circ}$. Each of the orbitals overlaps s-orbital of the hydrogen atom to form a covalent $\sigma$-bond. The structure of water molecule is presented in Figure 5. However, in water molecules an angle made by p-orbitals of the oxygen atom is $104.5^{\circ}$, which is $15^{\circ}$ greater than that displayed in Figure 5. This occurs because the $\mathrm{O}-\mathrm{H}$ bonds are not merely covalent. Their electron density is shifted towards oxygen. The latter acquires a surplus negative charge, and hydrogen atom acquires a positive one, which causes mutual repulsion of the orbitals and increases the angle. There is also an action of some other factors. Overall, the $\mathrm{H}_{2} \mathrm{O}$ molecule is a dipole having a positive charge on the one end and a negative charge on the other.

A hydrogen bond can form between $\mathrm{H}_{2} \mathrm{O}$ molecules if

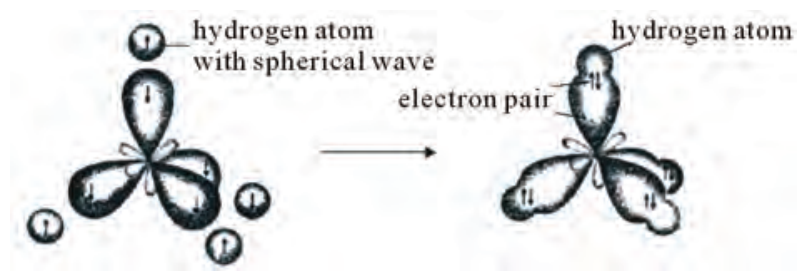

(a)

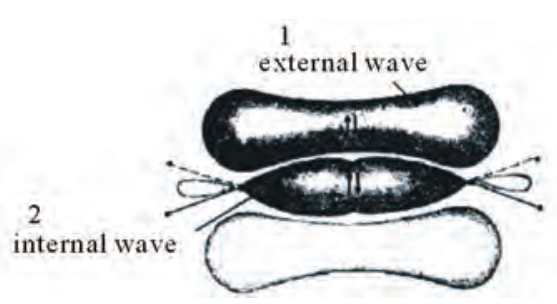

(b)

Figure 4. Two different programs used for the formation of covalent bonds in two different chemical compounds: a formation of methane molecule: each of four hydrogen atoms donates its electron to form pairs with four electrons of carbon residing in the lobe waves; $b$ - formation of ethylene molecules; 1 - top view of the plane where atoms are located, 2 - double bond waves (side view).

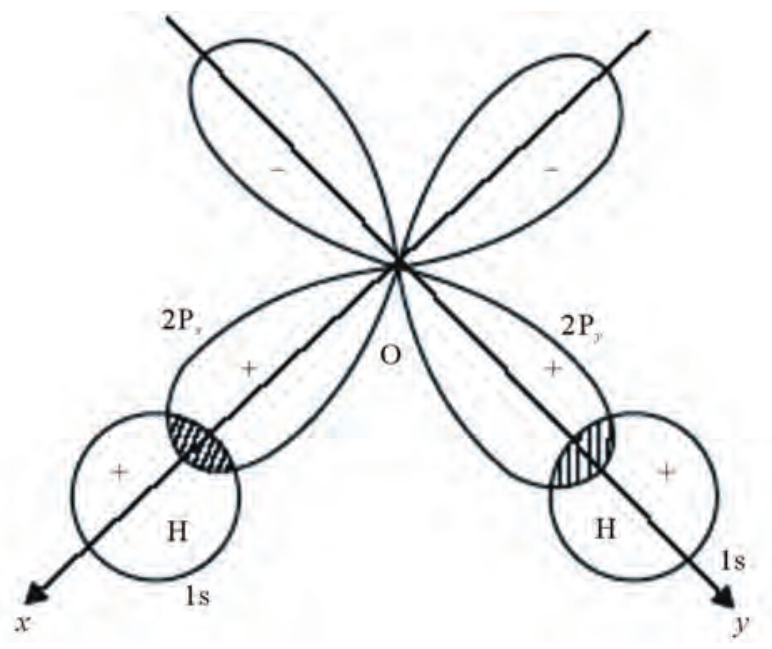

Figure 5. Bond formation in $\mathrm{H}_{2} \mathrm{O}$ molecule with participation of 2p-orbitals of the oxygen atom.

1s-orbital of the hydrogen atom is unoccupied. As we have seen, this condition is partially satisfied in $\mathrm{H}_{2} \mathrm{O}$ molecules. When approaching the second $\mathrm{H}_{2} \mathrm{O}$ molecule, its negative pole further shifts the electron cloud towards its "own" oxygen. The probability that the undivided electron pair of another $\mathrm{H}_{2} \mathrm{O}$ molecule with reside at the hydrogen atom is not high, although some exchange interaction is allowable. This makes a contribution to the hydrogen bond energy. The second (probably more significant) contribution is made by electrostatic interaction between hydrogen atoms (protons) and negatively charged oxygen atoms of other molecules.

Water plays a unique role in living objects. In human, it constitutes $70 \%$ of the body weight. All biochemical reactions take place in a water medium.

Thus, chemical determinate systems gave rise to natural diversity in inorganic, organic and bioorganic chemistry, and resulted in the formation of liquid and many solid crystals. This created prerequisites for origin of life on the Earth.

From a physical standpoint, the evolution of material word is based on increasing negentropy, i.e., the degree of orderliness. E. Schrodinger defined it as

$$
-S=k \lg (1 / D)
$$

where $-S$ is the negative entropy, or negentropy; $k$ is the Boltzmann's constant equal to $3.2983 \times 10^{-24} \mathrm{cal} / \mathrm{deg} ; D$ is the quantitative measure of disorderliness of atoms in the system, $\lg (1 / D)$ is the negative logarithm of $D$, and $1 / D$ is the measure of orderliness.

However, of prime importance for us is that increasing negentropy is always supported by increasing amount of structural information. This can be expressed by the following equation:

$$
-S=k \lg (1 / D)+\sum p_{i} \times \log p_{i}
$$


where $p_{i}$ is the probability of individual events in the system. Thus, the informational component in this equation determines an increase of negentropy in the system and is related with acquisition of new properties.

Developing the concept about a correspondence between negentropy and structural information, we can present the following equality:

$$
-S=k \lg (1 / D)=\sum p_{i} \cdot \log p_{i}
$$

Hence,

$$
\begin{gathered}
\lg (1 / D)=\left(\sum p_{i} \cdot \log p_{i}\right) / k, \text { and } \\
1 / D=10^{\left(\sum p_{i} \log p_{i}\right) / k} \text {, then } \\
D=10^{-\left(\sum p_{i} \log p_{i}\right) / k} \text { or } 1 / 10^{\left(\sum p_{i} \log p_{i}\right) / k}
\end{gathered}
$$

If in the Helmholtz equation for free energy entropy is replaced by $D$, this gives the following expression:

$$
F=U-\frac{T}{10^{\sum p_{i} \log p_{i} / k}}
$$

Thus, $F$ can be considered as a function of the amount of structural information in a system. This equation is essential for understanding the evolution processes.

New chemical structures (compounds) based on chemical determinate systems are still emerging in the nature. Now it is related with human activity aimed at the synthesis of various novel compounds that were earlier absent. However, the nature made an important step toward synthesis of high-polymer bioorganic compounds, such as proteins, nucleic acids, complex carbohydrates and lipids. Many of sporadically synthesized proteins could have unique enzymatic properties, thus making the synthesis processes more specific. After creation of this material possessing versatile chemical properties, the nature could move to the next step in systemic organization of material world, which led to a multiform world of living systems. This was another level of self-organization based on homeostatic determinate systems and using new memory elements and new action programs.

\section{Formation of Homeostatic Determinate Systems in the Living Nature}

The appearance of developed forms of life on the Earth was preceded by a long period of prebiological chemical evolution. Active self-organization and self-assembly of the structures with different complexity took place; this was accompanied by the mechanism of natural selection. The indicated processes occurred mainly in the "primary water bouillon". In terms of synergetics, these events were considered in detail by Haken [2], Prigogine [11], Eigen [12] and other authors.
To form a new type of homeostatic determinate systems able to provide further evolution of life on the Earth, another memory element was necessary for the creation of new determinate systems with new action programs. Such elements underlying the new memory mechanisms were found. Now they are called the genetic memory.

DNA molecules appeared quite suitable for this purpose. They are the double helices composed of desoxyribose, residues of phosphoric acid, and four types of nitrous bases - adenine, guanine, cytosine and thymine. Desoxyribose molecules, bound through phosphoric acid, form long polymeric chains. In DNA molecules, two such polymeric chains are connected to each other via nitrous bases attached to the first carbon atom of desoxyribose. Nitrous bases form the complementary pairs: adenine - thymine and guanine - cytosine.

They can be used to encode more than 20 features. These features are amino acids, which constitute any proteins. By the coding theorem, if an event can be presented on the average as $n$-binary digits, it can be symbolized also by any other events that admit encoding with $n$-binary digits, i.e.

$$
S_{v} n_{v} \geq S_{x} n_{x},
$$

where $S_{x}$ denotes the numbers of real events;

$S_{y}$ is the numbers of symbolic events;

$n_{x}$ and $n_{y}$ are the average number of digits, respectively, for one real and one symbolic event.

Let $x$ stand for amino acids, and $y$ for nucleotides. Then,

$$
\begin{aligned}
& n_{x}=\log _{2}^{20}=4.322, \\
& S_{x}=1(1 \text { amino acid }), \\
& n_{y}=\log _{2}^{4}=2, \\
& \qquad S_{y}=\frac{1 \times 4.322}{2}=2.161,
\end{aligned}
$$

which means that a minimum amount of nucleotides needed to symbolize any amino acid is 2.161, i.e., more than two and less than three. Encoding amino acids by nucleotide triplets, we obtain possible combinations of 4 elements taken 3 at a time, so that each combination unambiguously defines each event. Evidently, there are 20 combinations:

$\begin{array}{llll}\text { ABA } & \text { BCA } & \text { ADC } & \text { BDD } \\ \text { ABB } & \text { BCB } & \text { ADD } & \text { CDA } \\ \text { ACA } & \text { BCC } & \text { BDA } & \text { CDB } \\ \text { ACB } & \text { ADA } & \text { BDB } & \text { CDC } \\ \text { ACC } & \text { ADB } & \text { BDC } & \text { CDD }\end{array}$

As follows from the above reasoning, from the mathematical standpoint it is not impossible to encode the entire multiform sequence of protein amino acids using four nitrous bases.

Four types of nitrous bases gave rise to a triplet code. Each triplet of nitrous bases corresponds to one of the 
known 20 amino acids.

Thus, in the entire diversity of different macromolecules, DNA proved to be most appropriate for the formation of the next type of memory called the genetic memory. It is essential that DNA can split into two complementary chains and copy itself with the aid of DNApolymerase. It means that genetic information is completely retained at cell division and transferred from cell to cell, i.e., hereditarily. In a cell, three types of RNA (messenger, transfer and ribosomal) convey the genetic information written in DNA to different intracellular proteins, thus determining their specific structural and functional properties. This unique mechanism transforms genetic sign into phenotypic. In this mechanism an important role belongs to complementarity of nitrous bases, their ability to form the complementary pairs: adenine thymine and guanine - cytosine.

In the cell nucleus, DNA and histone proteins form a close-packed structure - chromatin. Upon cell division, histones are destroyed via hydrolysis and DNA is deproteinized. Free DNA is copied. This is followed by synthesis of histones, and two daughter DNA are packed again to form chromatin. All these events take place due to self-assembly.

Thus, self-assembly may occur between two type polymers: nucleic acids and proteins. This is exemplified by disassembly and self-assembly of tobacco mosaic virus (TMV) under laboratory conditions. RNA isolated from TMV and viral proteins spontaneously form the intact particles of a high virulence.

A particle of bacteriophage T4 has a complicated structure. It includes a head consisting of proteins, which has a shape of elongate icosahedron. The head is attached to a tail surrounded with a retractive cover. The latter is connected with a basal plate having six protein fibers. DNA resides in the head. This particle can be completely disassembled and assembled in a tube. A similar selfassembly occurs in a bacterial cell, where comes only the bacteriophage DNA containing the information about all its components. Copies of DNA and viral proteins are produced in a cell, this is followed by self-assembly of the virus particles, which then leave a cell, thus destructting it. Here, genetic information about the structure of viral macromolecules is supplemented with the structural information obtained due to self-assembly of particles, where other memory elements represented by chemical forces manifest themselves. This is how the nature interrelates evolutionary recent mechanisms of matter selforganization with evolutionary earlier mechanisms.

Certainly, biological objects with various complexity of their system organization are based on a large data base related with the formation and operation of physical and chemical determinate systems - this is a wide set of biological elements entering the structure of many enzymes, hormones, vitamins, where their known physiccal-chemical properties are used. In particular, iron is known to contribute to binding and transfer of oxygen by erythrocyte hemoglobin. Copper performs essential functions in the chain of electron transfer in mitochondria. Iodine is a necessary component of thyroid hormones. Cobalt enters the structure of vitamin $\mathrm{B}_{12}$. Calcium in phosphates forms the structure of bone tissue. $\mathrm{Na}^{+}$and $\mathrm{K}^{+}$define the electric potential of cell membranes. Electrolytes $\left(\mathrm{Na}^{+}, \mathrm{K}^{+}, \mathrm{Ca}^{2+}\right.$ and $\mathrm{Mg}^{2+}$ salts) determine osmotic blood pressure. Examples of the chemical elements involvement in complex biological systems are quite numerous.

Genetic memory underlay the formation of biological determinate systems of various complexity: morphofunctional determinate systems (cells, locomotor apparatus, vascular and nervous systems, etc.); homeostatic determinate systems (energy, water-salt, temperature homeostasis and many other systems responsible for constancy of internal medium of the organism).

Here we consider the principles of self-organization of energy homeostasis as a determinate system at the molecular level. ATP is consumed in different parts of the organism: for secretion of various substances, for membrane transport, biosynthesis, regeneration, physical work, heat generation, etc. In this connection, a certain determinate system should be active in the organism to restore the ATP content in a cell and integrally in the entire organism. This function is performed by energy homeostasis. Its structural-functional organization is shown in Figure 6.

This system has several levels of organization: cell level, organ level, and integral organism level. Result of its action is the ATP concentration in any cell of the biological system. ATP serves as the system-forming factor (factor of homeostasis). A similar notion is the degree of energization of adenylic nucleotides, or the energy charge. The bulk of ATP in a cell forms due to phosphorylation in the oxidation chain localized in mitochondria; and a minor part, due to substrate phosphorylation. The latter route is essential only at the oxygen deficiency. The oxidation processes in mitochondria are preceded by difficult transformations of energy substrates in the corresponding metabolic routes. For carbohydrates this is glycolysis (glycogenolysis). For fats, $\beta$-oxidation of fatty acids. A decay of one molecule of glucose yielding lactate reduces two molecules of NAD. One cycle of $\beta$-oxidation reduces respectively a molecule of NAD and FAD. Starting from acetyl-CoA, which forms both from carbohydrates and fats, the oxidation takes a common route: via the Krebs cycle and the oxidation chain of mitochondria. The reduction of three NAD molecules 


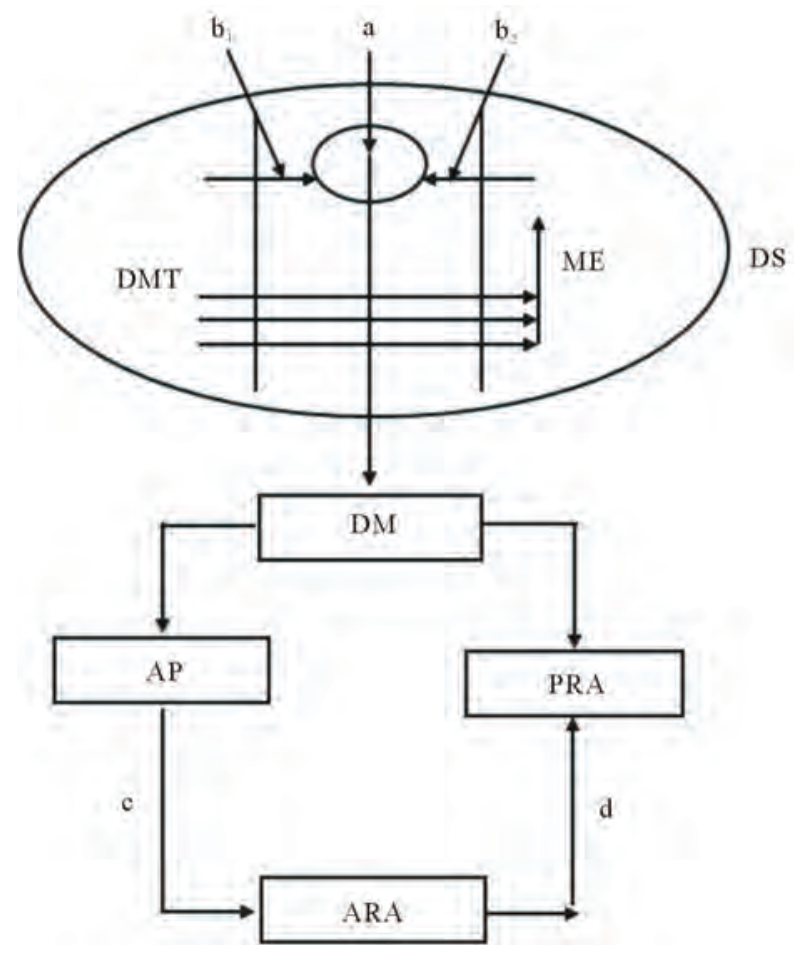

Figure 6. Energy homeostasis as a determinate system. DMT - dominant motivation: initial hormonal background of the organism (concentration of catecholamines, glucocorticoids, insulin, glucagon, etc.); ME - memory elements: gene pool of the target organs, four types of nitrous bases (adenine, thymine, guanine, cytosine); a - trigger signaling: stress (production of hormones able to interact with ME); b1 and b2 - circumstantial signaling (conditioning factors): nutrition pattern, changes in the emotional sphere, changes in the gene pool (mutations), action of pharmacological preparations and biologically active substances; DM - decision making: switching over the energy metabolism from carbohydrate type to the lipid one; AP - action program: energy metabolism of the organism; ARA - actual results of action: ATP concentration in cells; PRA - predicted result of action: the concentration ratios of substances in a cell directly affecting the ATP formation (ATP/ADP+Pn, $\left.\mathrm{NAD} / \mathrm{NAD} \cdot \mathrm{H}^{+}, \mathrm{FAD} / \mathrm{FAD} \cdot \mathrm{H}^{+}\right)$; DS - determinate synthesis; c and $\mathrm{d}-$ straight line and feedback.

and one FAD molecule corresponds to each cycle. We do not present the complete balance equations here. The oxidation of $\mathrm{NADH}^{+}$and $\mathrm{FAD} \cdot \mathrm{H}^{+}$occurs with the participation of oxidation chain in mitochondria. Overall, these processes can be considered as a general program of ATP regeneration in a cell. The mechanism of ATP formation requires a continuous delivery of ADP and Pn. Their pool in a cell is restored again at ATP decay.

Thus, a change in the concentration ratio of ATP/ADP + Pn serves as the first results-of-action acceptor in a cell. It determines the notion of phosphate potential (PP), or the Klingenberg potential. At rest, high energization of mitochondria and decreased content of ADP and Pn in- hibit the process of oxidative phosphorylation. On the contrary, in operative condition an increased content of ADP and Pn enhances it. Rate ratio of these processes is such that PP always tends to a certain constant value.

The second essential results-of-action acceptor in energy homeostasis is a change in the concentration ratios of $\mathrm{NAD} / \mathrm{NAD} \cdot \mathrm{H}^{+}$and $\mathrm{FAD} / \mathrm{FAD} \cdot \mathrm{H}^{+}$or, more precisely, in redox potential of the system. It is closely related with the value of phosphate potential. Its decrease at intense decay of ATP accelerates the oxidation processes in mitochondria. However, an increase in the concentration of NAD and FAD changes redox potential of the whole system and creates prerequisites for the activation of cell energy metabolism. At increasing phosphate potential, the situation is inversed. Thus, the concentration ratios of ATP/ADP $+\mathrm{Pn}^{+}, \mathrm{NAD} / \mathrm{NAD} \cdot \mathrm{H}^{+}$and $\mathrm{FAD} / \mathrm{FAD} \cdot \mathrm{H}^{+}$, serving as the results-of-action acceptor, are the essential units of intracellular regulation of energy metabolism. The action program is connected with the results-of-action acceptor via redox potential of the system (cell).

However, it is not indifferent to the entire organism which substrates in which tissues (organs) are to be oxidized under functional stress. Under acute short-term stress the oxidation of carbohydrates predominates in various tissues. Under chronic long-term stress two programs are formed: some tissues (organs), such as brain and erythrocytes, proceed with the oxidation of carbohydrates, i.e. implement the first program; other tissues, such as muscles and heart, switch over to the oxidation of fatty acids, i.e., implement the second program. Taking into account that muscular tissue constitutes more than a half of the body weight, one may say that the entire organism shifts its energy metabolism from carbohydrate type to the lipid one. This is confirmed by increasing blood content of lipids, decreasing respiratory coefficient, and changes in other parameters. Examples of such states include starvation, adaptation to cold, prolonged and quite intensive physical work. It is very important that even the brain can be shifted to the oxidation of lipids (ketone bodies). This is connected with the organization of energy homeostasis at the organ level.

Decision making concerning the use of some or other genetic program is based on determinate synthesis. Recall, it includes dominant motivation, conditional signaling, causal signaling, and memory. In this case, dominant motivation is determined by a change in the hormonal background. At acute functional tension of the organism, mainly the urgent adaptation mechanisms operate. They are related chiefly with an increased production of catecholamines. Rapid mobilization of a carbohydrate reserve (due to glycogen decay in liver and muscles under the action of phosphorylase) increases the blood content of glucose. In this phase, commonly the insulin content 
also increases. Favorable conditions for enhanced carbohydrate metabolism are established, which is the program number one.

A carbohydrate reserve in the organism is not large, so under prolonged stress the second genetic program is formed, leading to the oxidation of fats. This occurs due to changes in the dominant motivation caused by an increased blood content of glucocorticoids (cortisol) and a decreased content of insulin.

Along with the urgent mechanisms, the mechanisms of long-term adaptation also come into action (with a certain time shift). In fatty tissue, the fat-mobilizing effect is enhanced. In liver, glycolysis is inhibited and glyconeogenesis is intensified. In the process, essential are not only such urgent mechanisms as allosteric regulation, but also the induction of enzyme synthesis or, on the contrary, its suppression. The first mechanism is related, for example, to glyconeogenesis enzymes; and the second one, to the key enzymes of glycolysis. In liver, synthesis of VLDL is enhanced. In this case, decision making is affected by the information extracted from the memory elements (a gene pool).

This forms the structural trace of adaptation. Thus, decision making reflects the extent of adaptive changes, their quantitative rather than only qualitative aspect.

Biologically active substances that come into the organism with foodstuffs of vegetable or zoogenic origin and with folk-medicine preparations may play an important role in conditional signaling. Well known is the group of vegetal adaptogens: ginseng, eleutherococcus, rhodazine, leuzea, etc. These preparations have a broad spectrum of action, including also the energy metabolism; they facilitate mobilization of carbohydrates, free fatty acids, etc., exert gonadotropic effect, and improve non-specific resistance of the organism. Such pharmacological preparations as phenamine sharply enhance energy metabolism of the organism. All these substances act at the level of determinate synthesis, changing the mechanisms of both urgent and chronic adaptation.

Any extreme irritant (stress) can play a role of causal signalization for energy homeostasis. Its action forms a certain dominant motivation.

Cessation of an extreme irritant also serves as a signal for biological system. Homeostasis as a system persists, but moves to a new level of activity. Blood content of catecholamines and glucocorticoids decreases and content of insulin increases. Dominant motivation is altered; a different decision is made for initiating another (former) genetic program. However, the action of extreme irritant has left a structural trace. Due to induction, synthesis of many enzymes has changed. Immediate return to the initial state is impossible. Organism needs a time to "erase" this structural trace using the lysosomal enzymes. If the action of extreme irritant is repeated, the structural trace of adaptation will be more pronounced. Thus, decision making in homeostatic determinant systems does not proceed as a heuristic search, it is genetically determined and evolutionary reinforced by natural selection. Telenomy in homeostatic systems has an evolutionary solution.

Switching over the energy metabolism from carbohydrate type to the lipid one is characterized not only by quantitative changes in the oxidation ratio of carbohydrate and lipid substrates, but also by qualitative shifts. This relates primarily to rearrangement of respiratory chain in mitochondria, where peroxide oxidation of fatty acids and other substrates conjugated to phosphorylation becomes essential now.

All the features of energy metabolism in individual tissues (organs) are strictly coordinated at the level of whole organism. The same objective - formation of a necessary ATP amount - is attained differently in different tissues. Energy homeostasis works as a complex co-operative system. Such a cooperative mechanism provides the optimal conditions for enhancing the fat-mobilizing effect and FFA transport to muscles. When the organism is stressed, liver supplies nerve tissue and erythrocytes with carbohydrates due to activation of glyconeogenesis, and simultaneously delivers lipids to muscles due to activation of VLDL synthesis; thus, it operates according to programs number one and two.

At the level of entire organism other integrative mechanisms operate, different from those working in a cell. A particularly large number of feedforwards and feedbacks are functioning in the sphere of neuroendocrine regulation. Of basic importance are the endocrine-metabolic interrelations. Our works revealed that glucocorticoids increase the blood content of VLDL as well as the synthesis of apoproteins. In their turn, by the negative feedback mechanism, VLDL inhibit steroidogenesis in adrenal glands and decrease the production of glucocorticoids. VLDL are the main transport form of endogenic fat in the organism. As a transport form of the lipid nature energy substrates, VLDL are partially destructed in peripheral tissues under the action of lipoprotein lipase to form lipoproteins of a higher density, including HDL. The latter ones, being a supplier of the substrate (cholesterol) for steroid hormones, enhance steroidogenesis in adrenal glands. Such is the case with rats. In cattle, LDL serve as a source of cholesterol for steroidogenesis. Thus, changes in blood lipoprotein spectrum are the resultsof-action acceptor at the level of entire organism. Note, this is an essential but not sole example of feedback at the level of intrasystem and the more so of intersystem relations. In such a manner the energy homeostasis is operating, which we commonly call the energy metabolism. 
The ATP concentration in a cell is maintained due to free energy $(G)$ of chemical compounds, which are continuously oxidized during the operation of energy homeostasis.

Under prolonged functional stress, when carbohydrate and even lipid reserves become insufficient, the organism starts to consume its own proteins for energy production. The proteins are first hydrolyzed to amino acids, which are transformed into carbohydrates due to enhanced glyconeogenesis and then are oxidized into $\mathrm{CO}_{2}$ and $\mathrm{H}_{2} \mathrm{O}$ to yield ATP and a corresponding amount of heat. Thus, under stress the entropy of a biological system increases. There is a constant threat of equilibration with the environment. All homeostatic systems of the organism, including the energy homeostasis, work to counteract this treat.

Biological objects as hierarchical self-organized systems are tremendously more complicated than the objects of inanimate nature. However, their behavior under the action of external factors on the organism is fundamentally the same. For instance, in homoiothermal animals, constancy of the body temperature $\left(36.7^{\circ} \mathrm{C}\right)$ is maintained by temperature homeostasis. When the organism is exposed to low temperatures, its operation is rearranged. Under such conditions, fatty acids are oxidized intensely with a lower efficiency. A major part of energy is dissipated as heat. This decreases the weighted average body temperature. The system is rearranged in compliance with another genetic program (memory elements). The system goes to a new level of homeostasis; efficiency of the useful physical work decreases. As the ambient temperature is further lowered, a decrease in efficiency of the oxidation processes becomes more pronounced, and energy dissipation increases (the next level of homeostasis). Then the time comes when temperature homeostasis fails to perform its functions. This leads to progressive decrease in the body temperature. The organism is freezing.

Homeostasis maintaining the constancy of arterial pressure (AP) operates in a similar manner. In the norm, a ratio of maximum and minimum AP is $120 / 80 \mathrm{~mm} \mathrm{Hg}$. Meanwhile, stress triggers the release of adrenaline into the blood, thus raising the arterial pressure. The system moves to a new level of homeostasis. Then everything returns to the initial level. A permanent hypertension may occur in response to a prolonged increase of different pressure factors in the blood. The top values (levels of homeostasis) may vary depending on the organism. A human feels discomfort (headache, quick pulse). Life can be quite long at such level of homeostasis, until blood stroke or infarction comes. A decrease in osmotic or oncotic blood will lower AP. This leads to hypotension (another level of homeostasis). This is also not good, resulting in insufficient supply of oxygen and glucose to the organs (first of all, brain); lethal outcome cannot be excluded in a critical condition. Other homeostatic systems of the organism obey the same principle of operation. When the organism is exposed to external factors, homeostatic systems behave as dissipative ones, because additional energy is required to maintain homeostasis under extreme conditions.

The formation of homeostatic determinate systems in living objects is related with the appearance of internal medium of complex multicellular organisms. However, such organisms can perform various behavioral acts only if a new type of homeostatic determinate systems is present. These are the neurodynamic systems.

Memory elements in such systems are represented by nerve cells of cerebral cortex, which acquire information from both extero- and interoreceptors. Five senses correspond to exteroreceptors: vision, hearing, touch, smell and taste. Vision acquires information as electromagnetic modes. Hearing perceives it as acoustic vibrations. Touch senses tactile signals (sensations). Among them are also the temperature sensations. Smell and taste provide the organism with information in the form of chemical signals. The entire information comes to appropriate nerve centers called the analyzers. There are visual, auditory and tactile analyzers; smell and taste probably have a common analyzer. Information coming from internal receptors of the organism (interoreceptors) has no additional specificity and is perceived by the same analyzers. These are mainly chemical signals. Information conveyed to cerebral cortex is processed chiefly in vertical neuron chains that are perpendicular to the cortex surface. Thus, we have four types of central analyzers and the corresponding four types of neurons. The conceptions of external world and its changes are formed in central analyzers. Numerous interneuron contacts determine the connections of these parts of cerebral cortex with its locomotor parts, subcortical structures, limbic system, vegetative nervous system, etc.

Nervous activity is based on unconditioned and conditioned reflexes $[13,14]$. Conditioned reflexes are the attribute of high nervous activity. Neuromuscular apparatus intended for actualization of unconditioned reflexes should be referred to morphofunctional systems; and complex behavioral reactions of the organism based on numerous conditioned reflexes, to neurodynamic or psychophysiological determinate systems. Decision making and action program in such systems may be quite complicated. For example, a complex neurodynamic determinate system forms when a pilot strives to land a plane precisely on the runway (Figure 7). The goal-forming factor here is the necessity of pushing down the plane and superposing it with the runway. Landing conditions, which play a role of conditioning factor, may be very 
unfavorable: poor visibility, a strong crosswind, severe icing, etc. Using his knowledge of the plane control system and having an appropriate experience, the pilot coordinates his actions taking into account the aircraft position relative to the runway at each particular moment. Results-of-action acceptor here is the anticipated result superposition of the plane and the runway, although actual result of action may differ significantly from it. In such cases, pilot has to change the action program with the aid of a different decision making. When the task is accomplished, this neurodynamic determinate system ceases to be. However, in the period when the system exercised its functions, a large number of neurochemical connections operated to change the state of vegetative nervous, cardiovascular, respiratory, digestive, egestive and other systems of the organism. These mechanisms are thoroughly considered in the monograph by L. E. Panin [3].

The similarity of self-organization mechanisms in the living and inanimate nature is often accompanied by similar regularities of their behavior in the fields of external action. This refers to behavior of biological membranes as liquid crystals and behavior of solid crystals.

\section{Structural Transitions in Biological Membranes and Solid Crystals in Terms of Physical Mesomechanics and Thermodynamics}

Liquid and solid crystals are homeostatic determinate systems [3].

"Classical thermodynamics leads to the notion of system in equilibrium, such as, e.g., crystal” [4]. In solid crystals residing in the fields of weak external action, the state of homeostasis is determined by the nature of interatomic interactions. This is the metallic bonding, which has a quantum-mechanical nature. It is responsible for the high shear strength of crystals. According to the literature [15,

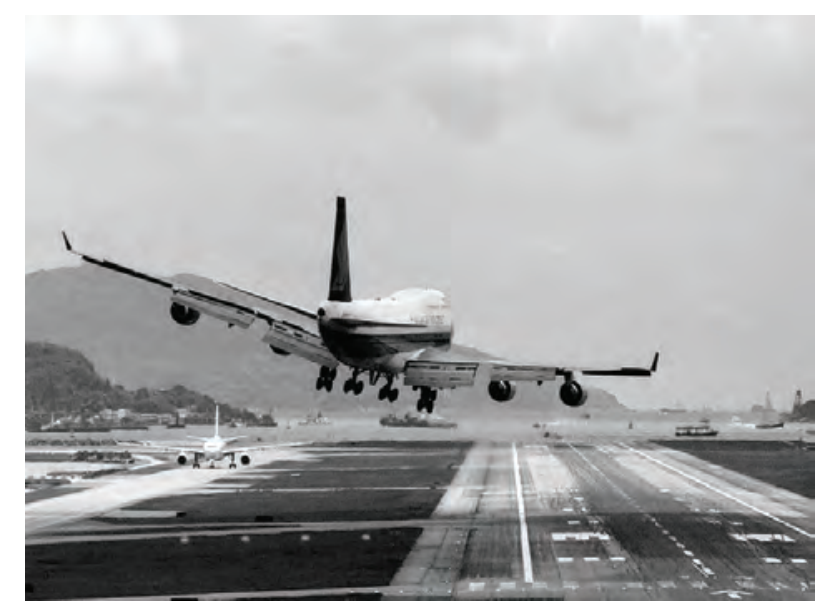

Figure 7. Landing in difficult conditions.
16], solid crystal in the fields of strong external action behaves as an open thermodynamically nonequilibrium system. "Pumping" of external energy results in a local loss of shear strength, which is accompanied by local structural-phase transformations in the crystal lattice. The possibility of such transformations is determined by electron energy spectrum of the crystal. This is confirmed by a relationship between packing-defect energy $(\gamma)$ of the crystal and its electronic structure, i.e., memory elements of the crystal $[3,17]$. In solid crystals, which are a structurally inhomogeneous medium, structural-phase transitions occur mainly on the external surface and at the internal interfaces. This is the place where a chessboard distribution of stresses and deformations is formed: the cells under compressive normal stress alternate chequerwise with the cells under tensile normal stress [18]. Tangential stresses also have a chessboard distribution, which is spatially displaced in phase by $\pi / 2$. This can explain the physical nature of plastic flow localization at different scale-structural levels under different conditions of loading. The excess molar volume and virtual nodes of a higher energy structure in the interstitial space that are present in the zones of tensile stress allow the occurrence of local structural transformations caused by nonequilibrium state in this zone.

A similar situation is observed for biological membranes under the action of various external factors (metal oxide nanoparticles, stress hormones). In biological membranes considered as liquid crystals, the system-forming bonds are represented by low-energy forces: covalent and hydrogen bonds, hydrophobic and weak electrostatic interactions. They serve as the memory elements and determine also a low shear strength of biological membranes and such notion as membrane "liquidness". It can be stated that homeostatic mechanisms of physical and biological systems in dissipative state are universal.

Destruction of solid and liquid crystals increases the molar volume.

A dependence of the Gibbs thermodynamic potential $\mathrm{F}(\mathrm{v})$ on the molar volume $\mathrm{v}$ taking into account local zones of different scale stress concentrators is described by the equation:

$$
F(v)=U-T S+p v-\sum \mu_{i} C_{i},
$$

where $\mu_{i}$ - chemical potential, $C_{i}-$ concentration (Figure 8, [15]) .

At critical values of molar volume $v_{i}=(1,2, \cdots, 6)$, the thermodynamic potential $F(v)$ has local minima. They reflect local nonequilibrium potentials in the zones of different scale hydrostatic tension. Critical values of $v_{I}$ correspond to different levels of homeostasis in a deformable solid:

$v_{0}$ is an equilibrium crystal; the initial level of homeo- 
stasis;

$v_{1}$ are the zones of stress microconcentrators where dislocation cores are generated; the next level of homeostasis;

$v_{2}, v_{3}$ are the zones of stress meso- and macroconcentrators where local structural-phase transitions with the formation of meso- and macrostripes of local plastic deformation take place; the next levels of homeostasis;

$v_{4}$ corresponds to intersection of curve $F(v)$ with the abscissa. At a further increase of the local molar volume, changes of the Gibbs thermodynamic potential proceed under the conditions of $F(v)>0$, and the system becomes unstable. Various forms of material failure appear; solid crystal starts to behave as a liquid one.

$v>v_{6}$ - the existence of two phases is possible: at $v=$ $v_{5}$ - the vacancy phase atom, at $v>v_{6}$ - different thermodynamic levels of the crystal lattice in a deformable solid, different levels of its homeostasis.

Thus, plastic deformation of solid and liquid heterocrystals in the fields of external action is a multilevel process of their destruction, with the corresponding levels of crystal lattice self-organization and levels of its homeostasis, i.e., the destruction via different phases of strengthening (self-organization). This decreases the orderliness and amount of structural information in the system.

Here we see the universal nature of homeostasis of biological and physical systems in dissipative state.

Dependence of Gibbs thermodynamic potential on the molar volume $v$ and changes in the structural information I), taking into account local zones of stress concentrators is determined by the expression:

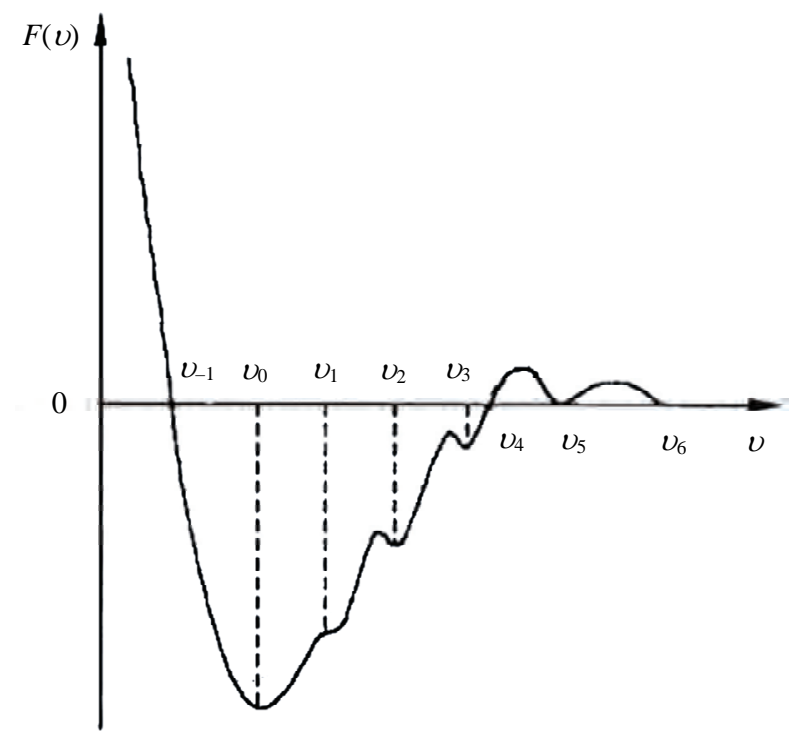

Figure 8. The dependence of the Gibbs thermodynamic potential $\boldsymbol{F}(v)$ from the molar volume $v$ in the light of local zones of stress concentrators of different scales [15].

$$
F(v, I)=U-T / 10^{\left(\sum p_{i} \log p_{i}\right) / k}+p v-\Sigma \mu_{i} C_{i}
$$

These quantitative interrelations underlie transition of the system to a new structural level of homeostasis.

The above changes can be adequately described in terms of determinate systems theory. Shock wave is the external signal specific to polycrystal (a multiscale structured medium). When a shock wave is passing through the sample, it interacts with the memory elements of the system (polycrystal). The memory elements are represented by different type connections stabilizing the structure in time (the initial level of homeostasis). In metals and alloys, these may be different manifestations of electrostatic interactions (ionic forces, metallic bonding, and Van der Waals forces). They are distributed nonuniformly over the volume of a deformed solid and vary in the energy level. Energy of these forces depends on the packing closeness of metal atoms or molecules in some alloys (intermetallides), the presence of defects (porosity) and impurities, and environmental influence (the action of temperature, magnetic field, etc.). In determinate systems theory, the latter is considered as the effect of conditioning factors that forms a background for the operation of system-specific external signals.

As a result of this interaction, a complex multilevel hierarchical system (crystalline structure) decides to choose some or other action program to be implemented. This may be the generation and development of rotational strain modes leading to rotational failure of the material (the first action program), or predominant generation of translational modes of deformation and appearance of shear bands leading to the formation of micro- and macrocracks (the second action program), or some other programs may be implemented.

In the process, the eventual result of action is attained, and a system (metal sample or construction material) moves to a new level of homeostasis. If the action of system-specific external signal persists, a new decision is made, and the system goes to the next level of homeostasis. This proceeds until the system (sample or construction) is completely destroyed. Such a mechanism was presented earlier as the structural levels of deformation of solids [19], and later as physical mesomechanics of deformable solids [20,21].

Results-of-action acceptor in metals and alloys or in deformable solids is represented by the atoms of a crystal lattice or by various mesostructures on which the action of new forces or their combination is closed. They stabilize the entire system in a new functional state at a different level of homeostasis (Figure 2).

These notions of determinate systems and their transition to a new level of homeostasis agree well with the modern ideas concerning the deformation mechanism of 
solids [22]. Structural levels of deformation refer to mesoscale levels and differ quailtatively from the single-level approach employed by continuum mechanics and dislocation theory. The novel approach revealed general regularities of the behavior of deformable solids at mesolevel and made it possible to use the synergy principle for explanation of self-consistent plastic flow at different mesoscale levels. This resulted in the development of multilevel mechanics of deformable solids, which was called the "physical mesomechanics" [21].

However, here we encounter the same problem as that indicated by W. Heisenberg in quantum physics [23]. The mathematical apparatus used to describe plastic deformation of solids is adequate for the process mechanics, whereas the language describing the physical phenomena is not adequate. The application of conceptual apparatus of determinate systems theory allows us to overcome this disagreement.

However, in biological membranes as liquid crystals, destruction is related with structural transitions and is generally accompanied by increasing structural orderlyness (the order $\rightarrow$ order transition). Earlier [24], it was shown that the action of steroid hormones on erythrocyte membranes disturbs the mechanisms of self-organization that operate in the cells in normal functional condition. The active $\mathrm{CO}, \mathrm{NH}$ and $\mathrm{OH}$ groups of stress hormones interact with $\mathrm{CO}$ and $\mathrm{NH}$ groups both of proteins and phospholipids in biological membranes. This leads to the formation of complex protein-lipid clusters, where "compressive” hydrophobic interactions are reinforced. Molecularly bound water is displaced to adjacent regions. Here, hydrostatic forces increase the "tensile" tangential stresses. Mobile nanostructural boundaries are formed, along which the biological membranes are destructed. This results in the formation of numerous pores and mesostrips of plastic deformation. In terms of physical mesomechanics, these transformations resemble those developing in solid crystals in the fields of external action (Figure 9). However, in biological membranes such self-organization may be related even with increasing order and decreasing entropy, but this is incompatible with conditions that determine cell viability. Structural transitions cover the membrane-bound enzymes, transmembrane carriers and hormone receptors. It is reasonable to say that cell membranes go to a new level of homeostasis (self-organization) which is incompatible with life. The nature of life implies dynamics. The cell dies. Here, one can tell about thermodynamic features related with changes in the structure and function (properties) of solid crystals and biological membranes in the fields of external action.

Various structural transitions (phase transitions, nanostructural, etc.) strongly contribute to the functional activity of a cell. These are the transitions like smectic A

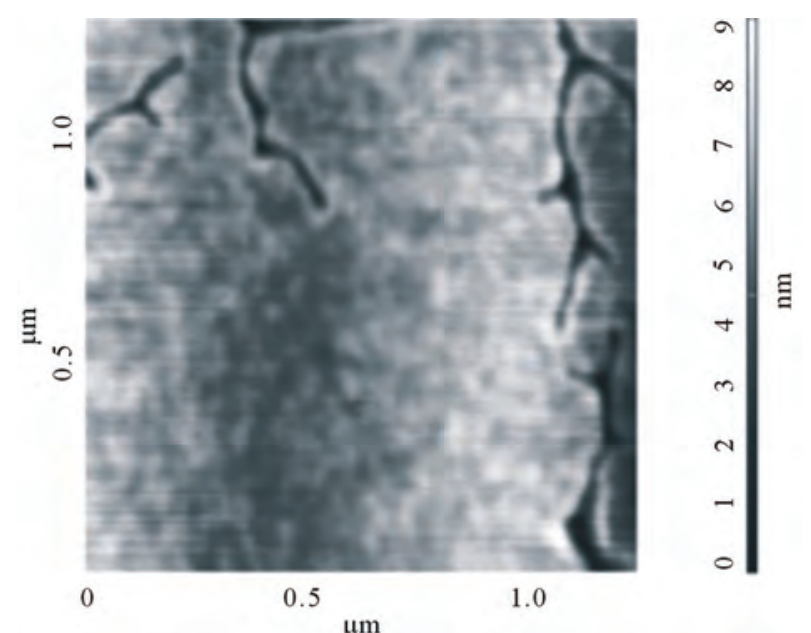

(a)

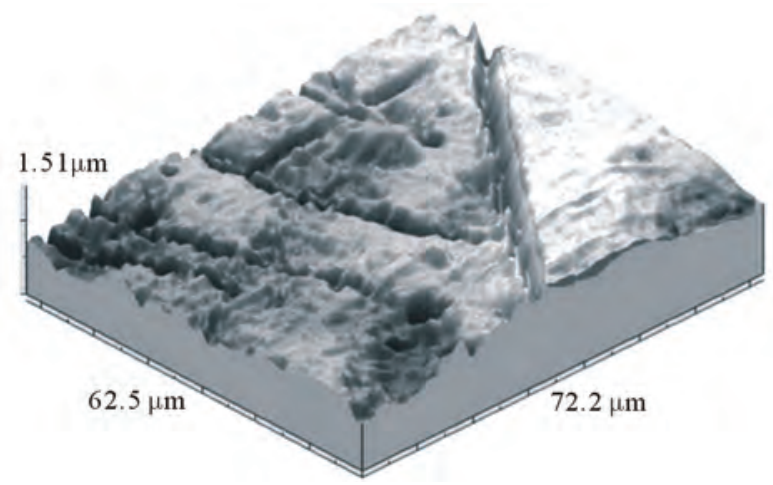

(b)

Figure 9. a - Atomic force microscopy. The surface of rat erythrocytes after adsorption of cortisol. Concentration of the hormone is $10^{-6} \mathrm{M}$. Deep meso-bands with bifurcation are seen; $\mathbf{b}$ - Formation of micropore chains along localized-deformation shear-bands. Plate of high-pure aluminum $180 \mathrm{~nm}$ thick glued on flat specimen of commercial Al. Alternative bending, $T=293 \mathrm{~K}$; cycle number $N=17.55 \times$ $10^{6}$ [15].

$\rightarrow$ smectic C, smectic $\rightarrow$ cholesteric, and nematic $\rightarrow$ isotropic state; in proteins, the transitions tangle $\rightarrow \beta$ structure and tangle $\rightarrow \alpha$-helix. They all affect the vital characteristics of a cell. I. Prigogine believed that there is "a wonderful analogy between instability of nonequilibrium origin and phase transitions" [4]. This problem is of great interest and deserves special examination.

\section{Conclusion}

Determinate systems represent a general principle of matter self-organization in the nature, from atom to human (Homo sapiens). Using the wave properties of elementary particles (first of all electrons), four quantum numbers characterizing their state, and Pauli exclusion 
principle, the nature created a remarkable diversity of physical and chemical phenomena. The first step towards increasing diversity was the formation of atoms and then the chemical elements. Today we know a little more than 100 chemical elements. Hydrogen is the first and most simple of them. Fermium takes the hundredth place. It is followed by mendelevium, nobelium, lawrencium, and some other not very stable elements. This exhausts the natural diversity associated with the structure of atoms. D. I. Mendeleev was the first to perceive that properties of the elements are in a periodical dependence on the charge of their atomic nuclei. In the present-day periodic table of chemical elements, atomic number, atomic weight, nuclear charge and physic-chemical properties are closely interrelated. However, the latter ones depend to a considerable extent on the changes in wave characteristics of the elementary particles constituting these atoms.

Eventually, everything is reduced to the quantum mechanical phenomena in atoms as complex homeostatic determinate systems connected with the outer world, i.e., physical vacuum. In these systems, memory elements are represented by four types of interaction: strong, electromagnetic, weak and gravitational. The action of these forces determines the nature of quantum interactions in any atom and hence its physic-chemical properties. The presence of unpaired valence electrons in the outermost energy levels increases the chemical diversity. The variation of electron wave properties has a tremendous potential. Electrons may form spherical waves, octuple waves, lobe waves, etc.; they may occupy different energy levels. At synthesis of chemical compounds, electrons generate various hybrid waves. Here the nature widely uses the chemical bonds. However, along with chemical bonds, other forces also contribute into chemical diversity. These are the ionic and hydrophobic interactions as well as hydrogen bonds.

The four type forces play a role of memory elements in chemical determinate systems. Transition to this level of systemic organization of material world led to a sharp amplification of natural diversity, which is now investigated by inorganic, organic and bioorganic chemistry. This type of systems includes also a multifarious world of solid and liquid crystals, which are considered by physics, geochemistry and biology.

In this case, an increase of negentropy in the course of evolution has the same meaning as accumulation of information, and is determined by Shanon equation:

$$
-H=\sum p_{i} \cdot \log p_{i}
$$

In open systems, increasing negentropy due to energy supply from the environment, always increases the probabilities of individual events determining the probability of a state of the entire system. This leads to ap- pearance of thermodynamically stable systems and determines the time's arrow moving toward decreasing entropy.

When biological determinate systems are formed, an increase in negentropy, structural information and natural diversity is based on the coding principles. They underlie the operation of genetic mechanisms in each vegetal or animal cell. In such systems, memory elements are represented by four types of nitrous bases: adenine, thymine, guanine and cytosine. This produced a large set of proteins with different properties: structural proteins, contractile proteins, enzymes, carriers, hormone receptors, etc. A tremendous variety of life forms on the Earth is based on these processes.

However, the appearance of intuitive and conscious forms of behavior in the objects of living nature became possible only after the genesis of central and peripheral nervous systems. They formed a basis for unconditioned and conditioned reflexes as well as developed neuron networks. The latter served as a substrate for higher nervous activity, including complex human behavior, consciousness, thinking and memory. Complicated forms of selforganization in material world evolutionarily derived from the earlier forms.

Therefore, self-organization of material world developed toward increasing the orderliness (negentropy), structural information and natural diversity due to free energy of the environment via the formation of homeostatic determinate systems and hierarchy of their interacttion.

\section{References}

[1] S. Hawking, "From Big Bang to Black Holes. A Brief History of Time,” Mir, Moscow, 1990.

[2] H. Haken, "Advanced Synergetic Instability Hierarchies of Self-Organizing Systems and Devices,” Mir, Moscow, 1985.

[3] L. E. Panin, "Determinate Systems in Physics, Chemistry and Biology," Siberian University Publishing House, Novosibirsk, 2008.

[4] G. Nicolis and I. Prigogine, "Self-Organization in NonEquilibrium Systems. From Dissipative Structures to Order through Fluctuations,” Mir, Moscow, 1979.

[5] F. Capra, “The Web of Life,” Publishing House Helios, Moscow, 2002

[6] E. Schrodinger, "What is Life from a Physical Standpoint?” Izdatel'stvo Inostrannoy Literatury, Moscow, 1972.

[7] L. E. Panin and V. E. Panin, "Effect of the 'Chessboard' and Mass Transfer in Interfacial Media of Organic and Inorganic Nature,” Physical Mesomechanics, Vol. 10, No. 6, 2008, pp. 5-20.

[8] D. Perkins, “Inside a Proton,” In: J. Malvy, Ed., Fundamental Structure of Matter, Mir, Moscow, 1984, pp. 
130-172.

[9] A. Einstein, “Omnibus Edition,” AN SSSR, Moscow, Vol. 3, 1967, p. 626.

[10] J. Silk, "The Big Bang. The Creation and Evolution of the Universe,” Mir, Mosco, 1980.

[11] I. Prigogine and S. I. Stenger, "Order out of Chaos," Bantam, New York, 1984.

[12] M. Eigen and P. Schuste, "The hyper-cycle. A principle of natural self-organization,” Mir, Moscow, 1982.

[13] I. M. Sechenov, "Selected Works," AN SSSR, Moscow, 1952.

[14] I. P. Pavlov, “Omnibus Edition,” AN SSSR, MoscowLeningrad, Vol. 1-6, 1951-1952.

[15] V. E. Panin and V. E. Egorushkin, "Nonequilibrium Thermodynamics of a Deformed Solid as Multilevel System. Corpuscular-Wave Dualism of Plastic Shear," Physical Mesomechanics, Vol. 11, No. 2, 2008, pp. 9-30.

[16] A. A. Shaniavski, "Modeling of Fatigue of Metals. Synergetics for Aviation," Publishing House of Scientific and Technical Literature, Ufa, 2007.

[17] V. E. Panin, E. F. Dudarev and L. S. Bushnev, "Structure and Mechanical Properties of Substituted Solid Solutons,” Metallurgy, Moscow, 1971.

[18] L. E. Panin and V. E. Panin, "Effect of the 'Chessboard' and Mass Transfer in Interfacial Media of Organic and Inorganic Nature,” Physical Mesomechanics, Vol. 10, No.
6, 2007, pp. 5-20. doi:10.1016/j.physme.2007.06.001

[19] V. E. Panin, Yu. V. Grinyaev, T. F. Elsukova and A. G. Ivanchin, "Structural Levels of Deformation of Solids," Russian Physics Journal, Vol. 25, No. 6, 1982, pp. 5-27.

[20] V. E. Panin, "Fundamentals of Physical Mesomechanics,” Physical Mesomechanics, Vol. 1, No. 1, 1998, pp. 5-22.

[21] V. E. Panin and Yu. V. Grinyaev, "Physical Mesomechanics as a New Paradigm at the Interface of Physics and Mechanics of a Deformable Solid," Physical Mesomechanics, Vol. 6, No. 4, 2003, pp. 9-36.

[22] V. E. Panin and L. E. Panin, "Scale levels of homeostasis in a deformable solids,” Physical Mesomechanics, vol. 7, no. 3-4, May-August 2004, pp. 5-20.

[23] W. Heisenberg, "Remarks on the origin of the relations of uncertainty,” In: W.C. Price and S.S. Chissick, Eds., The uncertainty principle and foundations of quantum mechanics, John Wiley \& Sons, London, New York, NY, 1977, pp. 3-6.

[24] L. E. Panin and V. E. Panin, "Thermodynamics and mesomechanics of nanostructural transition in biological membranes under stress," Proceedings of 12th International Conference of Mesomechanics "Multiscaling of synthetic and natural system with self-adaptive capability”, G. C. Sih and C. K. Chao, Eds., Taipei, Taiwan, June 2010, pp. 189-190. 